\title{
Modal decomposition for measuring the orbital angular momentum density of light
}

\author{
Christian Schulze ${ }^{a}$, Daniel Flamm $^{a}$, Angela Dudley ${ }^{b}$, Andrew Forbes ${ }^{b}$, and Michael Duparré $a$ \\ ${ }^{a}$ Institute of Applied Optics, Abbe Center of Photonics, Friedrich Schiller University Jena, \\ D-07743 Jena, Germany; \\ ${ }^{b}$ Council of Scientific and Industrial Research, National Laser Centre, P.O. Box 395, 0001 \\ Pretoria, South Africa.
}

\begin{abstract}
We present a novel technique to measure the orbital angular momentum (OAM) density of light. The technique is based on modal decomposition, enabling the complete reconstruction of optical fields, including the reconstruction of the beam's Poynting vector and the OAM density distribution. The modal decomposition is performed using a computer-generated hologram $(\mathrm{CGH})$, which allows fast and accurate measurement of the mode spectrum. The CGH encodes the modes of interest, whose powers and relative phase differences are measured from the far-field diffraction pattern of the incident optical field with the hologram transmission function. In combination with a classical measurement of Stokes parameters, including a polarizer and a quarter-wave plate in front of the hologram, the polarization state of each mode is measured. As a consequence, any arbitrary vector field can be reconstructed, including amplitude, phase, and polarization. Having all information on the optical field, the Poynting vector and the OAM density can be calculated directly. We applied our method to beams emerging from optical fibers and free space beams, which allows us to investigate arbitrary coherent mode superpositions with complexly shaped intensity and polarization distributions. As a reference distinct beams of known OAM density were created using a spatial light modulator (SLM). Comparisons of measured and expected results reveal very good agreement.
\end{abstract}

Keywords: orbital angular momentum, OAM, singular optics, modal decomposition, polarization, vortex.

\section{INTRODUCTION}

Laser beams carrying orbital angular momentum (OAM) have attained a lot of interest in the recent times. ${ }^{1-13}$ Such beams exhibit a rotating phase structure and consequently rotate in space during propagation. By transfering their momentum, they are able to spin microscopic particles and have hence received attention in the field of optical trapping and particle manupulation. ${ }^{14-17}$ Moreover, their unique properties have opened new opportunities in nonlinear optics and quantum optics, enabling the entanglement of single photons in a multistate system and hence picturing future applications of performing logical operations and free space communication with OAM states. ${ }^{12,18,19}$ The fast development of science focusing on beams carrying OAM was sped up by the ease of generating such beams using spiral phase plates or appropriate holograms that easily transform a simple Gaussian beam into a beam with helical phase structure of tunable OAM. ${ }^{12,20}$ Even fibers have been studied to generate OAM carrying beams in a controlled manner. ${ }^{21}$ Despite the lot of work that has been spend to generate these beams and to investigate their nature to search for new physical effects and applications, only a few approaches concentrate on the detection of the OAM. ${ }^{2-28}$ Regarding the measurement of the spatially resolved OAM density, only a single approach based on azimuthal decomposition is currently known. ${ }^{29,30}$ Even though this technique has the advantage of being independent of the scale of the beam, it necessitates to scan the beam in radial direction, which makes this approach time-consuming.

In this work we focus on a different approach to measure the OAM density of light, which stands out due its simplicity of the experimental setup and the ability to perform real-time experiments. The presented technique is based on the modal decomposition with correlation filters ${ }^{31}$ from which the optical field is reconstructed, hence providing all necessary information to infer Poynting vector and OAM density.

Further author information:

Christian Schulze: email: christian.schulze@uni-jena.de, Telephone: +49 (0)3641 947666

Complex Light and Optical Forces VII, edited by Jesper Glückstad, David L. Andrews, Enrique J. Galvez, Proc. of SPIE Vol. 8637, 863719 - @ 2013 SPIE · CCC code: 0277-786X/13/\$18 · doi: 10.1117/12.2004263 


\section{MODAL DECOMPOSITION}

Modal decomposition is a powerful tool for detailed investigation of laser fields. In laser sources the optical field that is generated is quantized into modes, e.g., considering a laser resonator or optical fibers. The principle of the modal decomposition is to determine the power content and relative phase of the modes that compose the beam. Mathematically, any optical field can be expressed in terms of orthogonal basis functions:

$$
\boldsymbol{U}(\boldsymbol{r})=\sum_{l=1}^{2 N} c_{l} \mathbf{\Psi}_{l}(\boldsymbol{r}),
$$

with $\boldsymbol{r}=(x, y)$ the spatial coordinates, $c_{l}=\varrho_{l} e^{i \varphi_{l}}$ the complex expansion coefficient, $\mathbf{\Psi}_{l}(\boldsymbol{r})$ the $l^{\text {th }}$ vector mode with amplitude $\varrho_{l}$ and phase $\varphi_{l}$ (with respect to a reference phase) and $N$ the number of modes. The beam intensity is easily reconstructed from the modal amplitudes and phases via

$$
I(\boldsymbol{r})=|\boldsymbol{U}(\boldsymbol{r})|^{2} .
$$

The modal decomposition of a laser field as described by Eq. (1) can be performed all-optically using correlation filters. ${ }^{31,32}$ Such a filter performs a correlation of the incident field with the modes that are encoded into the filter, which allows to measure the power and relative phase of each individual mode. This ability is based on the specific design of the transmission function of the holographic device that constitutes the correlation filter. This transmission function depends on the quantity to be measured. For measuring the power of a distinct mode, the conjugate complex of that very mode is chosen to be the transmission function: ${ }^{31}$

$$
T_{l}(\boldsymbol{r})=\Psi_{l}^{*}(\boldsymbol{r}),
$$

where "*" denotes the complex conjugation. Using this transmission function, the intensity on the optical axis in the Fourier plane of the correlation filter is $\propto \varrho_{l}^{2}$.

To measure the phase difference of a mode to a chosen reference mode, two transmission functions, each representing an interferometric superposition of two modes, are necessary: ${ }^{31}$

$$
T_{l}^{\cos }(\boldsymbol{r})=\left[\Psi_{0}^{*}(\boldsymbol{r})+\Psi_{l}^{*}(\boldsymbol{r})\right] / \sqrt{2} \text { and } T_{l}^{\sin }(\boldsymbol{r})=\left[\Psi_{0}^{*}(\boldsymbol{r})+i \Psi_{l}^{*}(\boldsymbol{r})\right] / \sqrt{2}
$$

Again, from the intensities $I_{l}^{\text {sin }}$ (corresponding to $T_{l}^{\sin }$ ) and $I_{l}^{\cos }$ (corresponding to $T_{l}^{\cos }$ ) on the optical axis in the Fourier plane of the correlation filter, the phase angle can be calculated unambiguously according to ${ }^{31}$

$$
\Delta \varphi_{l}=-\arctan \left[\frac{2 I_{l}^{\sin }-\varrho_{l}^{2}-\varrho_{0}^{2}}{2 I_{l}^{\cos }-\varrho_{l}^{2}-\varrho_{0}^{2}}\right]
$$

To measure modal amplitudes and phases simultaneously with one filter only, we use angular multiplexing, which means the final transmission function $T(\boldsymbol{r})$ of the hologram is a superposition of all single transmissions functions $T_{n}(\boldsymbol{r})$ (for each modal amplitude and sine and cosine of the phase), each multiplied with a certain carrier frequency $\boldsymbol{K}_{n}$, to achieve a spatial seperation of the information in the Fourier plane: ${ }^{31}$

$$
T(\boldsymbol{r})=\sum_{n=1}^{3 N-2} T_{n}(\boldsymbol{r}) e^{i \boldsymbol{K}_{n} \boldsymbol{r}} .
$$

Since by this approach all amplitudes and phases can be measured simultaneously, a high measurement rate is possible, which is immense advantage for many applications3. We demonstrated real-time measurements with up to $30 \mathrm{~Hz}$. This number is primarily limited by the frame rate of the used CCD cameras.

However, the correlation filter can only correlate scalar, i.e., linearly polarized, fields. In the general case of arbitrarily polarized beams, a complete description of the optical field $\boldsymbol{U}$ is provided by the subsequent analysis of the cartesian field components $U_{x}$ and $U_{y}$, including the proper phase difference $\delta$ between them.

This can be done by determination of the Stokes parameters $S_{0} \ldots S_{3}$ of the beam, which necessitates six 
(assuming completely polarized light) modal decomposition measurements with a quarter-wave plate and a polarizer in appropriate orientations in front of the hologram: ${ }^{33,34}$

$$
\boldsymbol{S}=\left[\begin{array}{c}
S_{0} \\
S_{1} \\
S_{2} \\
S_{3}
\end{array}\right]=\left[\begin{array}{c}
\left|U_{x}\right|^{2}+\left|U_{y}\right|^{2} \\
\left|U_{x}\right|^{2}-\left|U_{y}\right|^{2} \\
2\left|U_{x}\right|\left|U_{y}\right| \cos \delta \\
2\left|U_{x}\right|\left|U_{y}\right| \sin \delta
\end{array}\right]=\left[\begin{array}{c}
I\left(0^{\circ}\right)+I\left(90^{\circ}\right) \\
I\left(0^{\circ}\right)-I\left(90^{\circ}\right) \\
I\left(45^{\circ}\right)-I\left(135^{\circ}\right) \\
I_{\lambda / 4}\left(45^{\circ}\right)-I_{\lambda / 4}\left(135^{\circ}\right)
\end{array}\right],
$$

where $I(\alpha)$ is the measured (respectively reconstructed) intensity behind the polarizer at angular orientations $\alpha=0^{\circ}, 45^{\circ}, 90^{\circ}, 135^{\circ}$ and $I_{\lambda / 4}(\alpha)$ denotes two additional measurements with the polarizer placed at $\alpha=45^{\circ}$, $135^{\circ}$ and a preceding quarter-wave plate. By this means, the last equality relates theoretical and experimental definition of the Stokes parameters, which enables the calculation of the phase difference $\delta$ :

$$
\delta=\arctan \left(\frac{S_{3}}{S_{2}}\right)
$$

Performing the depicted six modal decompositions both transverse cartesian components of the optical field are determined. Hence, the information on the optical field is complete, since amplitude, phase, and polarization are measured. This abundance of information can be used for many applications, e.g., to determine physical quantities that are not easily accessible with other techniques, such as the OAM density.

\section{ORBITAL ANGULAR MOMENTUM DENSITY}

As shown in the previous section, the modal decomposition using a correlation filter yields the complete information about the optical field. Hence, the Poynting vector $\boldsymbol{P}$ can be calculated by ${ }^{35}$

$$
\mathbf{P}(\mathbf{r})=\frac{1}{2} \Re\left[\frac{i}{\omega \epsilon_{0}} \epsilon^{-1}(\mathbf{r})[\nabla \times \mathbf{U}(\mathbf{r})] \times \mathbf{U}^{*}(\mathbf{r})\right],
$$

for vector fields $\boldsymbol{U}$ and by ${ }^{36}$

$$
\mathbf{P}(\mathbf{r})=\frac{\epsilon_{0} \omega}{4}\left[i\left(U \nabla U^{*}-U^{*} \nabla U\right)+2 k|U|^{2} \boldsymbol{e}_{z}\right]
$$

for scalar fields $U$, where $\omega$ is the angular frequency, $\epsilon$ is the permittivity, $\epsilon_{0}$ is the permittivity of vacuum, $k=2 \pi / \lambda$ is the wave number, and $\boldsymbol{e}_{z}$ is the unit vector in $z$-direction. From the Poynting vector the OAM is easily accessible from ${ }^{37}$

$$
\boldsymbol{j}=\boldsymbol{r} \times \frac{\boldsymbol{P}}{c^{2}} .
$$

Hence, the OAM density is measurable for both, scalar and vector fields, using the modal decomposition principle as outlined in section 2. In the following the z-component $j_{z}$, which is of most relevance for application, is considered only and termed "OAM density".

\section{RESULTS}

\subsection{Laguerre-Gaussian beams}

To verify the procedure as described by section 2 and 3, Laguerre-Gaussian beams of known OAM density (calculable using Eq. (10) and (11)) were generated, which served as a reference to the modal decomposition results. To generate different test beams a phase-only pixelated Spatial Light Modulator (SLM) was used, which

was programmed using the coding technique of Arrizon et al. ${ }^{38}$ to convert complex transmission functions to phase-only phase functions. The SLM was embedded in an experimental setup as schematically depicted in Fig. 1 $\left(\mathrm{SLM}_{1}\right)$. A linearly polarized helium neon laser $(\lambda=633 \mathrm{~nm})$ was expanded by a telescope and illuminated the SLM to approximate a plane wave. The field generated with the SLM was filtered in its Fourier plane to enhance the quality of the beam and was relay imaged onto a second SLM $\left(\mathrm{SLM}_{2}\right)$, which was used as correlation filter. ${ }^{31}$ Together with a single lens and a $\mathrm{CCD}$ camera $\left(\mathrm{CCD}_{1}\right) \mathrm{SLM}_{2}$ performed the modal decomposition, which yields the OAM density. The modal decomposition was done by displaying the transmission functions of Eq. (3) and (4) on $\mathrm{SLM}_{2}$ subseqquently, and recording the correlation signal in the Fourier plane at $\mathrm{CCD}_{1}$, which yields the 


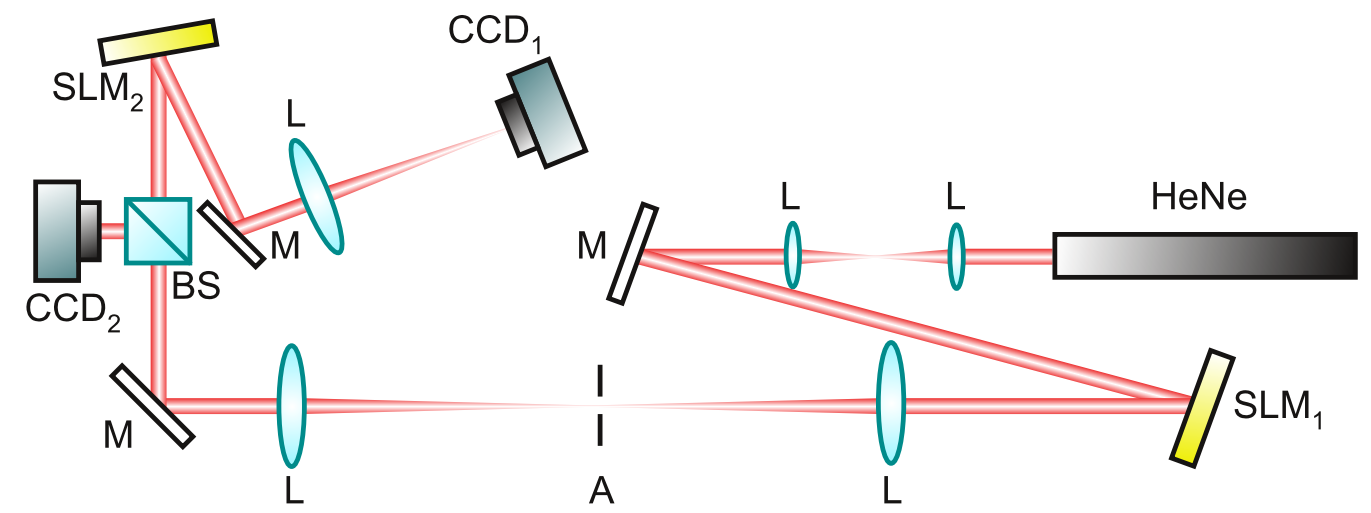

Figure 1. Experimental setup for the generation and characterization of Lagurre-Gaussian beams of different order. HeNe helium neon laser, L lens, $\mathrm{M}$ mirror, $\mathrm{SLM}_{1,2}$ Spatial Light Modulators, A aperture, $\mathrm{CCD}_{1,2}$ CCD cameras, BS beam splitter

modal powers $\varrho_{l}^{2}$ and phases $\Delta \varphi_{l}$, and hence, the optical field (Eq. (1)), the Poynting vector (Eq. (9), (10)), and the OAM density (Eq. (11)). To record the beam under test, a second CCD camera $\left(\mathrm{CCD}_{2}\right)$ and a beam splitter were used. As a first example a pure Laguerre-Gaussian mode $\mathrm{LG}_{p=0, l=1}$ was generated with the radial index $p$ and the azimuthal index $l$, whereas last mentioned index indicates the order of the helical phase and the topological charge, respectively. ${ }^{4,12}$ Fig. 2 depicts the results of the characterization of this beam. Fig. 2(a) shows the measured near field intensity, which reveals the typical donut shape with a hole in its center indicating the phase singularity. The results of the modal decomposition are pictured in Fig. 2(b) and (d). As expected, the modal power spectrum reveals a correlation signal for the $\mathrm{LG}_{0,1}$ mode only, whereas all other mode powers in the range $p=0 \ldots 2$ and $l=0 \ldots 2$ are zero. Additionally, all phases are measured to be zero. From the measurement of modal powers and phases the beam intensity is reconstructed according to Eq. (1) and (2), as shown in Fig. 2(c). The comparison of reconstructed (Fig. 2(c)) and measured beam intensity (Fig. 2(a)) reveals very good agreement. Applying the procedure of section 3 to the beam under investigation, the OAM density is inferred (Fig. 2(e)) and compared to the theoretical one (Fig. 2(f)). Both densities compare very well and resemble the intensity of the beam, which can be viewed as a characteristic of Laguerre-Gaussian beams and is a logical consequence of Eq. (10) and (11). Remarkably, the OAM density is exclusively positive. However, this fact can be easily understood, considering that the z-component of the OAM density is proportional to the $\varphi$-component of the Poynting vector, and hence, to the azimuthal index $l$ (here $l=+1$ ), provided the beam carries a helical phase. ${ }^{36}$ Moreover, it becomes clear that presented technique is capable of measuring the OAM density quantitatively. Here, the maximum is found with $2.5 \times 10^{-22} \mathrm{Ns} / \mathrm{m}^{2}$.
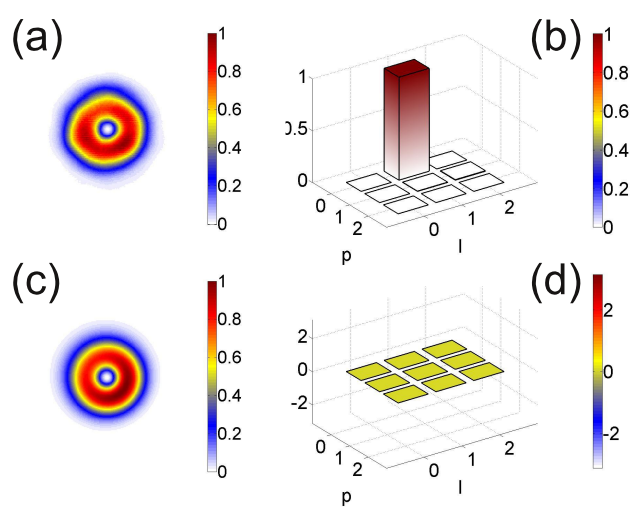

(e)

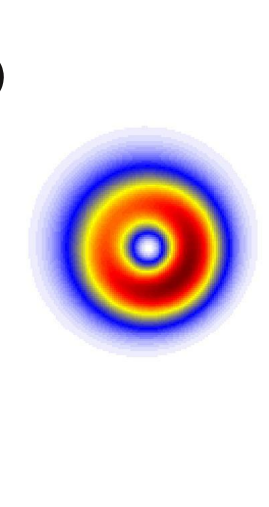

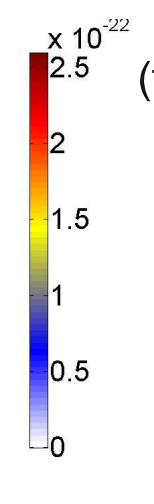

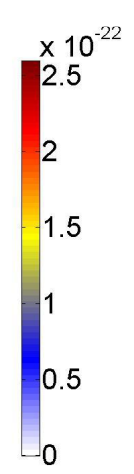

Figure 2. Characterization of a Laguerre-Gaussian $\mathrm{LG}_{0,1}$ mode beam. (a) Measured near field intensity. (b) Modal power spectrum. (c) Reconstructed intensity. (d) Modal phase spectrum. (e) Measured OAM density. (f) Calculated OAM density. OAM densities in $\mathrm{Ns} / \mathrm{m}^{2}$. 
(a)

(c)

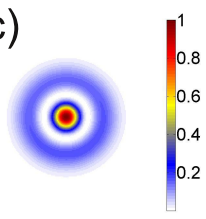

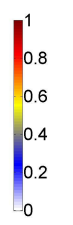

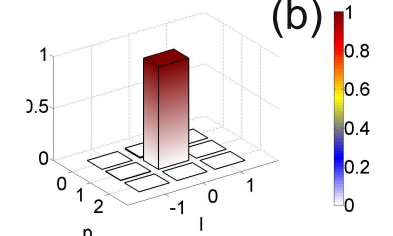

(d)

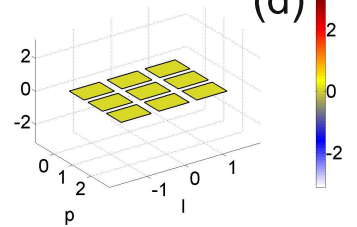

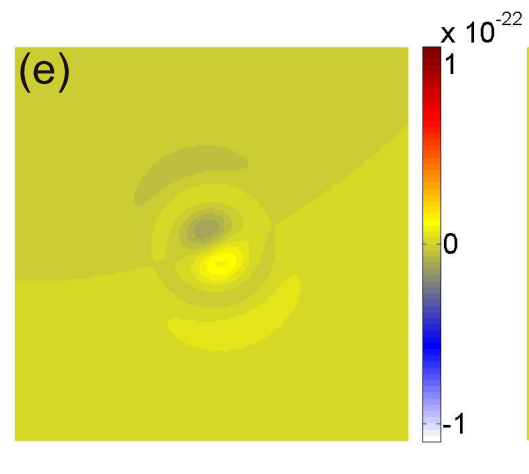

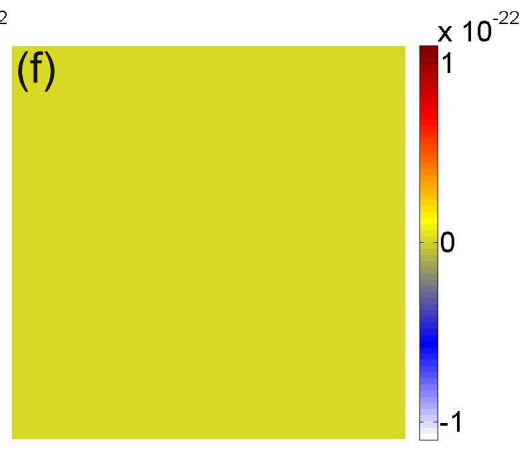

Figure 3. Characterization of a Laguerre-Gaussian $\mathrm{LG}_{1,0}$ mode beam. (a) Measured near field intensity. (b) Modal power spectrum. (c) Reconstructed intensity. (d) Modal phase spectrum. (e) Measured OAM density. (f) Calculated OAM density. OAM densities in $\mathrm{Ns} / \mathrm{m}^{2}$.

The results of Fig. 2 showed that a beam having a helical phase structure has a non-zero OAM density distribution, which mainly follows the intensity distribution. However, Laguerre-Gaussian beams with an azimuthal index $l=0$ will have a vanishing $\varphi$-component of the Poynting vector $P_{\varphi},{ }^{36}$ and consequently a vanishing OAM density. To proove this relation, a Laguerre-Gaussian mode $\mathrm{LG}_{1,0}$ was generated and characterized. The corresponding results are depicted in Fig. 3. The modal power spectrum in Fig. 3 reveals a single correlation signal at $p=1$ and $l=0$. Again, reconstructed and measured beam intensity correlate very well. Fig. 3(e) and (f) picture the measured and calculated OAM density, which is zero. Small deviations result from the intensity background of camera $\mathrm{CCD}_{1}$ used for the correlation measurement, which leads to a lower detection limit for the modal powers. Hence, the marginal content of other modes yields a slightly non-zero OAM density in the measurement. However, regarding the absolute scale it is obvious that peak value of the measured OAM density of a $\mathrm{LG}_{1,0}$ beam is more than one order of magnitude smaller than the one of the $\mathrm{LG}_{0,1}$ mode beam. Until now the investigated beams were single Laguerre-Gaussian modes. To provide a more complex example, we examined an in-phase superposition of two Laguerre-Gaussian beams $\mathrm{LG}_{2,1}+\mathrm{LG}_{0,-3}$, whose results are depicted in Fig. 4. Clearly, the modal power spectrum (Fig. 4(b)) reflects the existence of the two involved modes. From the phase spectrum it can be seen that both modes are in-phase. Again, reconstructed and measured intensity are in good agreement. Fig. 4(e) and (f) depict the measured and calculated OAM density, which correlate very well. Since the azimuthal indices of the involved modes are of positive and negative sign $(l=1$ and $l=-3)$, the resulting OAM density exhibits positive and negative values as well. Regarding the scale of the OAM density, the pure $\mathrm{LG}_{0,1}$ mode and the superposition $\mathrm{LG}_{2,1}+\mathrm{LG}_{0,-3}$ are of the same order of magnitude of $10^{-22} \mathrm{Ns} / \mathrm{m}^{2}$.

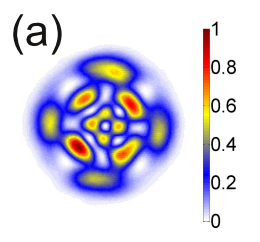

(c)

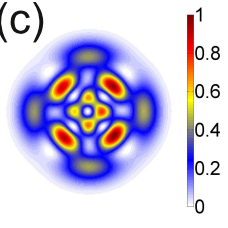

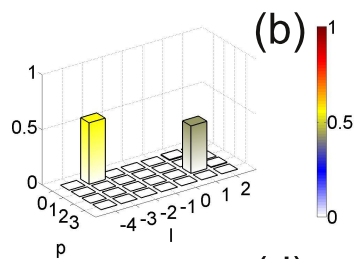

$\mathrm{p}$

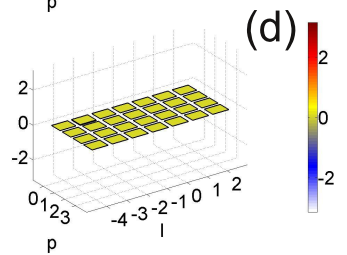

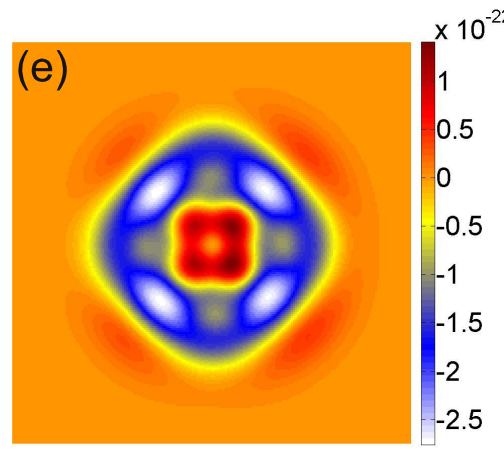

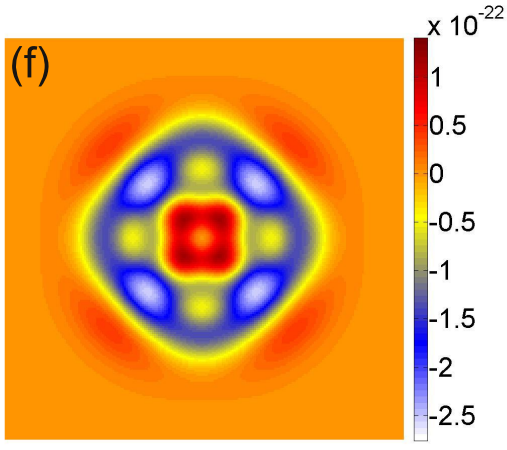

Figure 4. Characterization of a Laguerre-Gaussian $\mathrm{LG}_{2,1}+\mathrm{LG}_{0,-3}$ beam. (a) Measured near field intensity. (b) Modal power spectrum. (c) Reconstructed intensity. (d) Modal phase spectrum. (e) Measured OAM density. (f) Calculated OAM density. OAM densities in $\mathrm{Ns} / \mathrm{m}^{2}$. 


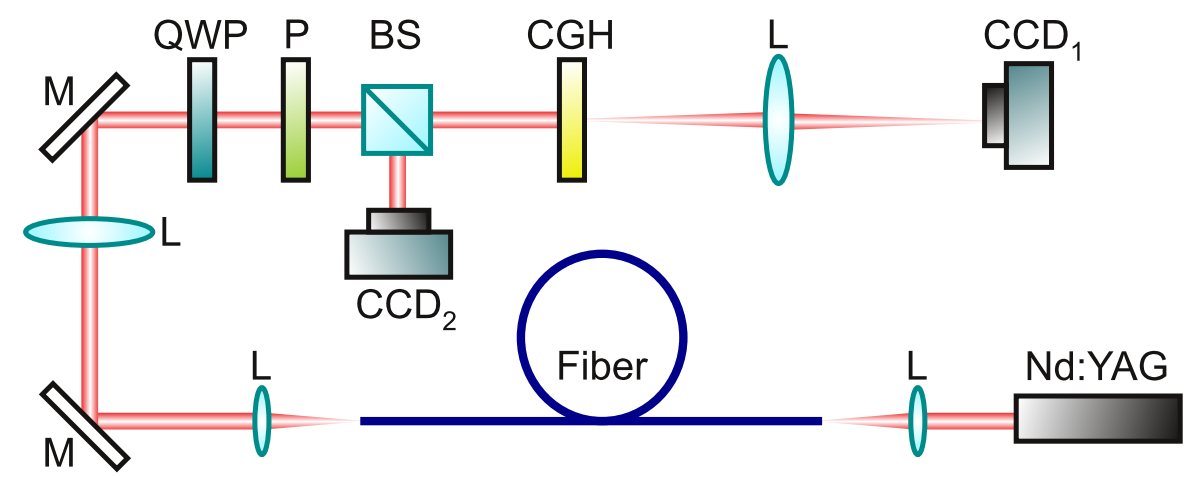

Figure 5. Experimental setup for measuring the OAM density of a fiber beam. Nd:YAG Nd:YAG laser, L lens, M mirror, QWP quarter-wave plate, $\mathrm{P}$ polarizer, BS beam splitter, CGH computer-generated hologram, CCD ${ }_{1,2} \mathrm{CCD}_{\text {cameras }}$

\subsection{Fiber beams}

The comparison of the measured OAM densities with the ones expected for the artifically generated reference beams proved the presented technique to yield accurate results regarding shape and absolute scale. Hence, the method can be applied to unknown laser beams, for which no reference can be provided. A suitable example is a beam emerging from an optical multimode fiber. Instead of programming modes and mode superpositions on a seperate SLM for beam generation, the optical field is here created naturally by multimode interference inside the fiber. Correspondingly, the experimental setup was changed according to Fig. 5. As beam source a multimode fiber (core diameter $25 \mu \mathrm{m}$, numerical aperture $\mathrm{NA}=0.064$ ), which guides six transverse modes, was seeded with the fundamental Gaussian beam of a Nd:YAG laser $(\lambda=1064 \mathrm{~nm})$. The fiber output was relay imaged (4f-imaged) to the correlation filter, which was in this experiment a static binary amplitude-only computer-generated hologram $(\mathrm{CGH})$, as used by Kaiser et al. ${ }^{31}$ Since such a filter is amplitude-only a different coding technique was used. ${ }^{39}$ To perform the polarization measurement, as described by Eq. (7), a quarterwave plate and a polarizer preceded the CGH. As in the previous experiment, a 2 f-setup behind the CGH (previously SLM) enabled the correlation measurement with a $\mathrm{CCD}$ camera $\left(\mathrm{CCD}_{1}\right)$. Again, a beam splitter in front of the hologram and a second $\mathrm{CCD}$ camera $\left(\mathrm{CCD}_{2}\right)$ provided the recording of the beam intensity. Fig. 6 illustrates an example of a fiber beam consisting of $27 \% \mathrm{LP}_{01}, 0 \% \mathrm{LP}_{02}, 41 \% \mathrm{LP}_{11 e}, 30 \% \mathrm{LP}_{11 o}, 2 \% \mathrm{LP}_{21 e}$, $0 \% \mathrm{LP}_{210}$. The comparison of measured (Fig. 6(a)) and reconstructed (Fig. 6(b)) beam intensity reveals very good agreement. Note that both intensities represent the sum of intensities recorded in $\mathrm{x}$ - and $\mathrm{y}$-direction of the polarizer. Fig. 6(c) illustrates the inferred OAM density of this beam. Apparantly, the OAM density is much larger than in the previous cases, with a maximum of $5 \times 10^{-12} \mathrm{Ns} / \mathrm{m}^{2}$. This is a logical consequence of the much smaller dimension of the beam, which is roughly extended within $30 \mu \mathrm{m}$ of diameter.

(a)

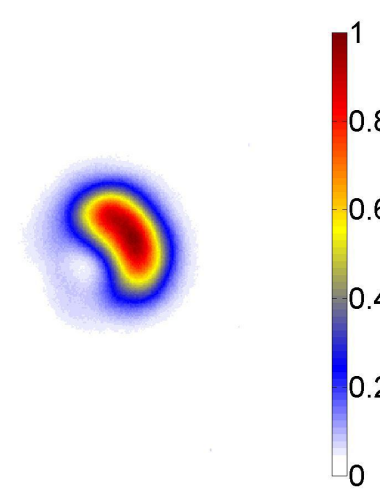

(b)

0.8

6

0.4

0.2

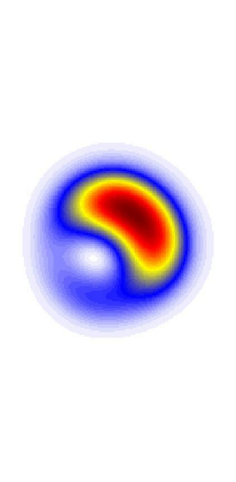

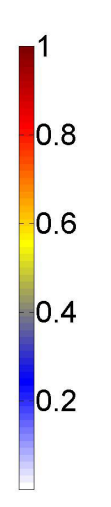

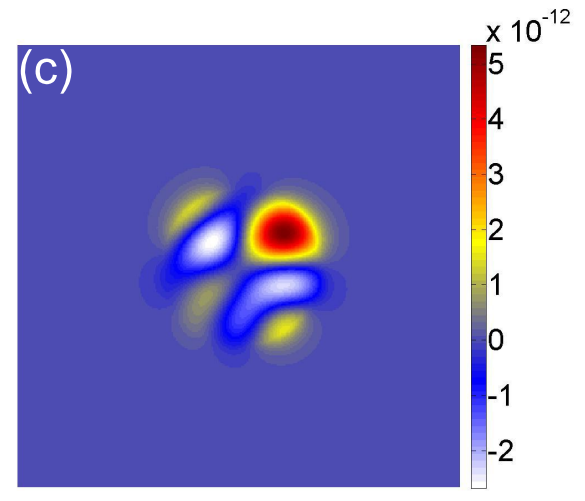

Figure 6. Characterization of a fiber beam consisting of a superposition of six modes $\left(27 \% \mathrm{LP}_{01}, 0 \% \mathrm{LP}_{02}, 41 \% \mathrm{LP}_{11 e}\right.$, $30 \% \mathrm{LP}_{11 o}, 2 \% \mathrm{LP}_{21 e}, 0 \% \mathrm{LP}_{21 o}$ ). (a) Measured near field intensity. (b) Reconstructed intensity. (c) Measured OAM density. OAM densities in $\mathrm{Ns} / \mathrm{m}^{2}$. 


\section{SUMMARY}

In summary, we demonstrated a novel measurement system to quantify the orbital angular momentum density of light. The technique bases on the well known correlation filters to perform a modal decomposition with subsequent reconstruction of the optical field, which allows to infer Poynting vector and the OAM density using a simple relationship. The method stands out due its easy setup, necessitating only an appropriate hologram, a lens and a camera, and its capability of real-time measurements. Linearly polarized Laguerre-Gaussian beams of different order were generated with a spatial light modulator and served as reference beams of known OAM density to prove the achieved results. Both, measured and theoretically expected OAM densities are in very good agreement, hence proving the accuracy of the technique. As an application case, the method was used to characterize a vector beam emerging from a multimode optical fiber. In consequence the correlation filter method is easily applicable to scalar and vector fields and allows the characterization of the OAM density in an easy and fast manner.

\section{REFERENCES}

[1] Allen, L., Beijersbergen, M. W., Spreeuw, R. J. C., and Woerdman, J. P., "Orbital angular momentum of light and the transformation of laguerre-gaussian laser modes," Phys. Rev. A 45(11), 8185-8189 (1992).

[2] Barnett, S. M. and Allen, L., "Orbital angular momentum and nonparaxial light beams," Opt. Commun. 110(56), $670-678$ (1994).

[3] Courtial, J., Dholakia, K., Allen, L., and Padgett, M., "Gaussian beams with very high orbital angular momentum," Opt. Commun. 144(4-6), 210 - 213 (1997).

[4] Soskin, M. S., Gorshkov, V. N., Vasnetsov, M. V., Malos, J. T., and Heckenberg, N. R., "Topological charge and angular momentum of light beams carrying optical vortices," Phys. Rev. A 56(5), 4064-4075 (1997).

[5] Padgett and Allen, "The angular momentum of light: optical spanners and the rotational frequency shift," Opt. Quant. Electron. 31, 1-12 (1999).

[6] Allen, L. and Padgett, M. J., "The poynting vector in laguerre-gaussian beams and the interpretation of their angular momentum density," Opt. Commun. 184(1-4), 67 - 71 (2000).

[7] O'Neil, A. T., MacVicar, I., Allen, L., and Padgett, M. J., "Intrinsic and extrinsic nature of the orbital angular momentum of a light beam," Phys. Rev. Lett. 88(5), 053601 (2002).

[8] Volke-Sepulveda, K., Garcés-Chávez, V., Chávez-Cerda, S., Arlt, J., and Dholakia, K., "Orbital angular momentum of a high-order bessel light beam," J. Opt. B: Quantum Semiclass. Opt. 4(2), 82 (2002).

[9] Padgett, M., Courtial, J., and Allen, L., "Light's orbital angular momentum," Physics Today 57(5), 35-40 (2004).

[10] Roux, F. S., "Distribution of angular momentum and vortex morphology in optical beams," Opt. Commun. 242(1-3), 45 - 55 (2004).

[11] Franke-Arnold, S., Allen, L., and Padgett, M., "Advances in optical angular momentum," Laser $\mathscr{G}$ Photon. Rev. 2(4), 299-313 (2008).

[12] Yao, A. M. and Padgett, M. J., "Orbital angular momentum: origins, behavior and applications," Adv. Opt. Photon. 3(2), 161-204 (2011).

[13] Belmonte, A. and Torres, J. P., "Digital coherent receiver for orbital angular momentum demultiplexing," Opt. Lett. 38(2), 241-243 (2013).

[14] Friese, M. E. J., Enger, J., Rubinsztein-Dunlop, H., and Heckenberg, N. R., "Optical angular-momentum transfer to trapped absorbing particles," Phys. Rev. A 54(2), 1593-1596 (1996).

[15] Garcés-Chávez, V., McGloin, D., Padgett, M. J., Dultz, W., Schmitzer, H., and Dholakia, K., "Observation of the transfer of the local angular momentum density of a multiringed light beam to an optically trapped particle," Phys. Rev. Lett. 91(9), 093602 (2003).

[16] Cojoc, D., Garbin, V., Ferrari, E., Businaro, L., Romanato, F., and Fabrizio, E. D., "Laser trapping and micro-manipulation using optical vortices," Microelectronic Engineering 78, 125 - 131 (2005).

[17] Zhao, Y., Shapiro, D., Mcgloin, D., Chiu, D. T., and Marchesini, S., "Direct observation of the transfer of orbital angular momentum to metal particles from a focused circularly polarized gaussian beam," Opt. Express 17(25), 23316-23322 (2009). 
[18] Gibson, G., Courtial, J., Padgett, M., Vasnetsov, M., Pas'ko, V., Barnett, S., and Franke-Arnold, S., "Freespace information transfer using light beams carrying orbital angular momentum," Opt. Express 12(22), $5448-5456$ (2004).

[19] Chu, S.-C., Ohtomo, T., and Otsuka, K., "Generation of doughnutlike vortex beam with tunable orbital angular momentum from lasers with controlled hermite-gaussian modes," Appl. Opt. 47(14), 2583-2591 (2008).

[20] Lee, W. M., Yuan, X.-C., and Cheong, W. C., "Optical vortex beam shaping by use of highly efficientirregular spiral phase plates for optical micromanipulation," Opt. Lett. 29(15), 1796-1798 (2004).

[21] Bozinovic, N., Golowich, S., Kristensen, P., and Ramachandran, S., "Control of orbital angular momentum of light with optical fibers," Opt. Lett. 37(13), 2451-2453 (2012).

[22] Beth, R. A., "Mechanical detection and measurement of the angular momentum of light," Phys. Rev. 50(2), 115-125 (1936).

[23] Berkhout, G. C. G., Lavery, M. P. J., Courtial, J., Beijersbergen, M. W., and Padgett, M. J., "Efficient sorting of orbital angular momentum states of light," Phys. Rev. Lett. 105(15), 153601 (2010).

[24] Berkhout, G. C. G., Lavery, M. P. J., Padgett, M. J., and Beijersbergen, M. W., "Measuring orbital angular momentum superpositions of light by mode transformation," Opt. Lett. 36(10), 1863-1865 (2011).

[25] Lavery, M. P. J., Berkhout, G. C. G., Courtial, J., and Padgett, M. J., "Measurement of the light orbital angular momentum spectrum using an optical geometric transformation," J. Opt. 13(6), 064006 (2011).

[26] Leach, J., Padgett, M. J., Barnett, S. M., Franke-Arnold, S., and Courtial, J., "Measuring the orbital angular momentum of a single photon," Phys. Rev. Lett. 88(25), 257901 (2002).

[27] Pires, H. D. L., Woudenberg, J., and van Exter, M. P., "Measurement of the orbital angular momentum spectrum of partially coherent beams," Opt. Lett. 35(6), 889-891 (2010).

[28] Wei, H., Xue, X., Leach, J., Padgett, M. J., Barnett, S. M., Franke-Arnold, S., Yao, E., and Courtial, J., "Simplified measurement of the orbital angular momentum of single photons," Opt. Commun. 223(1-3), $117-122(2003)$.

[29] Dudley, A., Litvin, I. A., and Forbes, A., "Quantitative measurement of the orbital angular momentum density of light," Appl. Opt. 51(7), 823-833 (2012).

[30] Litvin, I. A., Dudley, A., Roux, F. S., and Forbes, A., "Azimuthal decomposition with digital holograms," Opt. Express 20(10), 10996-11004 (2012).

[31] Kaiser, T., Flamm, D., Schröter, S., and Duparré, M., "Complete modal decomposition for optical fibers using CGH-based correlation filters," Opt. Express 17(11), 9347-9356 (2009).

[32] Flamm, D., Schulze, C., Schmidt, O. A., Schrter, S., and Duparr, M., "Mode analysis of lma fibers using the correlation filter method," in [Fiber Lasers IX: Technology, Systems, and Applications], Honea, E. C. and Hendow, S. T., eds., 8237, 8237OZ, SPIE (2012).

[33] Born, M. and Wolf, E., [Principles of Optics], Cambridge University Press, 7th edition ed. (1999).

[34] Berry, H. G., Gabrielse, G., and Livingston, A. E., "Measurement of the stokes parameters of light," Appl. Opt. 16(12), 3200-3205 (1977).

[35] Schulze, C., Naidoo, D., Flamm, D., Schmidt, O. A., Forbes, A., and Duparré, M., "Wavefront reconstruction by modal decomposition," Opt. Express 20(18), 19714-19725 (2012).

[36] Litvin, I. A., Dudley, A., and Forbes, A., "Poynting vector and orbital angular momentum density of superpositions of bessel beams," Opt. Express 19(18), 16760-16771 (2011).

[37] Aiello, A., Lindlein, N., Marquardt, C., and Leuchs, G., "Transverse angular momentum and geometric spin hall effect of light," Phys. Rev. Lett. 103(10), 100401 (2009).

[38] Arrizón, V., Ruiz, U., Carrada, R., and González, L. A., "Pixelated phase computer holograms for the accurate encoding of scalar complex fields," J. Opt. Soc. Am. A 24(11), 3500-3507 (2007).

[39] Lee, W.-H., "Binary computer-generated holograms," Appl. Opt. 18(21), 3661-3669 (1979). 